\title{
Efficient Processing of Multiple XML Twig Queries
}

\author{
Huanzhang Liu, Tok Wang Ling, Tian Yu, and Ji Wu \\ School of Computing, National University of Singapore \\ \{liuhuanz, lingtw, yutian, wuji\}@comp.nus.edu.sg
}

\begin{abstract}
Finding all occurrences of a twig pattern in an XML document is a core operation for XML query processing. The emergence of $\mathrm{XML}$ as a common mark-up language for data interchange has spawned great interest in techniques for filtering and content-based routing of XML data. In this paper, we aim to use the state-of-art holistic twig join technique to address multiple twig queries in a large scale XML database. We propose a new twig query technique which is specially tailored to match documents with large numbers of twig pattern queries. We introduce the super-twig to represent multiple twig queries. Based on the super-twig, we design a holistic twig join algorithm, called MTwigStack, to find all matches for multiple twig queries by scanning an XML document only once.
\end{abstract}

\section{Introduction}

Recently, XML has emerged as a standard information exchange mechanism on the Internet. XML employs a tree-structured model to represent data. XML query languages, such as XQuery and XPath, typically specify patterns with selection predicates on multiple elements for matching XML documents. Twig pattern matching has been identified as a core operation in querying tree-structured XML data.

Many algorithms have been proposed to match XML twig pattern $[3,7,8$, 11]. [3] decomposes the twig pattern into binary structural relationships, then matching the binary structural relationships and merging these matches. Bruno et al. [7] improved the methods by proposing a holistic twig join algorithm, called TwigStack. The algorithm can largely reduce the intermediate result comparing with the previous algorithms. Later on, Chen et al. [8] proposed a new Tag+Level labeling scheme and iTwigJoin algorithm to improve TwigStack. Lu et al. [11] designed a novel algorithm, called TJFast, which employed extended Dewey to match XML twig queries.

XML query processing also arises in the scenario of information dissemination, such as publish-subscribe (pub-sub) systems. In a typical pub-sub system, many user submitted profiles are presented by XPath expressions, and an XML document is presented as input. The goal is to identify the queries and their matches in the input XML document, and disseminate this information to the users who posed the queries $[4,9]$. 
In a huge system, where many XML queries are issued towards an XML database, we expect to see that the queries have many similarities. In traditional database systems, there have been many studies on efficient processing of similar queries using batch-based processing. Since pattern matching is an expensive operation, it would save a lot in terms of both CPU cost and I/O cost if we can process multiple similar twig queries simultaneously and only scan the input data once to get all the results. [6] has proposed Index-filter to process multiple simple XPath queries (no branch) against an XML document and it aims to find all matches of multiple simple path queries in an XML document. To eliminate redundant processing, it identifies query commonalities and combines multiple queries into a single structure. But Index-Filter does not consider how to process multiple twig queries.

Motivated by the recent success in efficient processing multiple XML queries, we consider the scenario of matching multiple XML twig queries with high similarity against an XML document.

The contributions of this paper can be summarized as follows:

- We introduce a new concept, called super-twig, which combines multiple twig queries into just one twig pattern. We also give the algorithm of constructing super-twig.

- Based on super-twig, we develop a new multiple twig queries processing algorithm, namely MTwigStack. With the algorithm, we can find all matches of multiple twig queries by scanning input data only once.

- Our experimental results show that the effectiveness, scalability and efficiency of our algorithm for multiple twig queries processing.

The rest of this paper is organized as follows. Preliminaries are introduced in Section 2. The algorithm MTwigStack is described in Section 3. Section 4 is dedicated to our experimental results and we close this paper by conclusion and future work in Section 5.

\section{Preliminaries}

\subsection{Data Model}

We model XML documents as ordered trees, each node corresponding to an element or a value, and the edges representing element-subelement or elementvalue relationships. Each node is assigned a region code (start: end, level) based on its position in the data tree $[3,6,7]$, start is the number in sequence assigned to an element when it is first encountered and end is equal to one plus the end of the last element visited, level is the level of a certain element in its data tree. Each text value is assigned a region code that has the same start and end values. Then structural relationships between tree nodes (elements or values), such as parent-child or ancestor-descendant, whose positions are labelled with region encoding can be determined easily. Figure 1 (a) shows an example XML data tree with region encoding. 


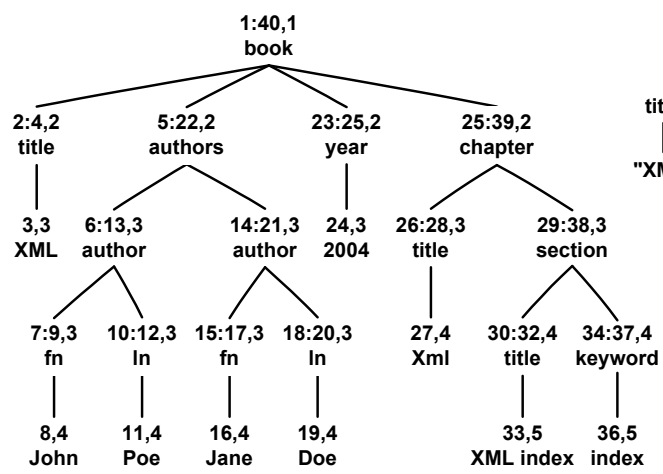

(a)

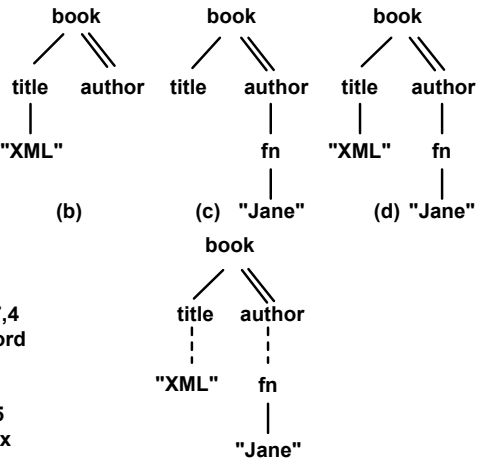

(e)

Fig. 1. An XML tree (a), three twig queries (b, c, d) and the super-twig query (e)

\section{$2.2 \quad$ Super-Twig Query}

When multiple twig queries are processed simultaneously, it is likely that significant commonalities between queries exist. To eliminate redundant processing while answering multiple queries, we identify query commonalities and combine multiple twig queries into a single twig pattern, which we call super-twig. Supertwig can significantly reduce the bookkeeping required to answer input queries, thus reducing the execution time of query processing. We will use $q$ (and its variants such as $q_{i}$ ) to denote a node in the query or the subtree rooted at $q$ when there is no ambiguity. We introduce the concepts OptionalNode and OptonalLeafNode to distinguish super-twig query from general twig queries.

In this paper, we only consider the tree patterns belonging to the fragment of XPath $X P^{\{/, / /,[]\}}[5]$ and the scenario that commonalities only existing in the top parts of the twigs. Given a set of twig queries against an XML document, $Q=\left\{q_{1}, \ldots, q_{k}\right\}$ belonging to $X P^{\{/, / /,[]\}}$, and assuming there is no repeated node in each query, we combine all the queries into a super-twig such that:

- The set of nodes in the super twig pattern equals the union of the sets of nodes of all individual twig queries;

- Each twig query is a subpattern (defined by [10]) of the super twig pattern;

- If the queries have different root nodes, we rewrite the queries whose root nodes are not the root of the XML document and add the document's root as the root node of the queries. Then the root node of the super twig pattern is same as the document's root;

- Suppose $n$ is a query node which appears in $q_{i}$ and $q_{j}, P_{i}$ and $P_{j}$ are the paths from the root to $n$ in $q_{i}$ and $q_{j}$ respectively, $P_{i}$ is same as $P_{j}$ and $m$ is the parent node of $n$ in these two queries. If the relationship between $m$ and $n$ is Parent-Child (P-C) in $q_{i}$, Ancestor-Descendant (A-D) in $q_{j}$, then the relationship between $m$ and $n$ in super-twig is relaxed to A-D;

- Suppose $n$ is a query node in one query $q_{i}$ of $Q$, and $m$ is the parent node of $n$ in $q_{i}$. Let $Q_{n}$ is the subset of twig queries of $Q$ which contain node $n$, and $Q_{m}$ is the subset of twig queries which contain node $m$ (the path from its 
root to $m$ must be a prefix of the path from its root to $n$ ). If $Q_{n} \subset Q_{m}$, we call $n$ an OptionalNode. And if all the relationships between $m$ and $n$ in $Q_{n}$ are P-C relationships, then the relationship between $m$ and $n$ in the super twig pattern is P-C (called optional parent-child relationship and depicted by a single dotted line); otherwise, the relationship between $m$ and $n$ in the super twig is A-D (called optional ancestor-descendant relationship and depicted by double dotted lines);

- Following the same situations of the above item and assuming $n$ is OptionalNode, let $Q_{x}=Q_{m}-Q_{n}$. If $m$ is a leaf node in some queries of $Q_{x}$ (so $\left.Q_{x} \neq \emptyset\right)$, then we call $m$ an OptionalLeafNode.

Example 1. In Figure 1, (e) shows the super-twig query of three queries (b), (c) and (d). "XML" and fn are OptionalNodes, title and author are OptionalLeafNodes. The edge which connects "XML" to title represents optional parent-child relationship. It means that we can output path solution "book-title" whether or not the element title has a child whose content is "XML" in an XML document, or output path solution "book-title-'XML'" when the element title has a child whose content is "XML" in an XML document.

\section{3 super-twig}

To combine multiple twigs into a super-twig, we should normalize them first. It means to obtain a unique XPath query string from a tree pattern sorting the nodes lexicographically. We use the method proposed in [12], for example, the normal form of $/ \mathrm{a}[\mathrm{q}][\mathrm{p}] / \mathrm{b}[\mathrm{x}[\mathrm{z}] / \mathrm{y}]$ is $/ \mathrm{a}[\mathrm{p}][\mathrm{q}] / \mathrm{b}[\mathrm{x}[\mathrm{y}][\mathrm{z}]]$. Then we design an algorithm according to the principles proposed in the last section, as shown in Algorithm 1. We input twig queries one by one and output the super-twig presented by XPath query.

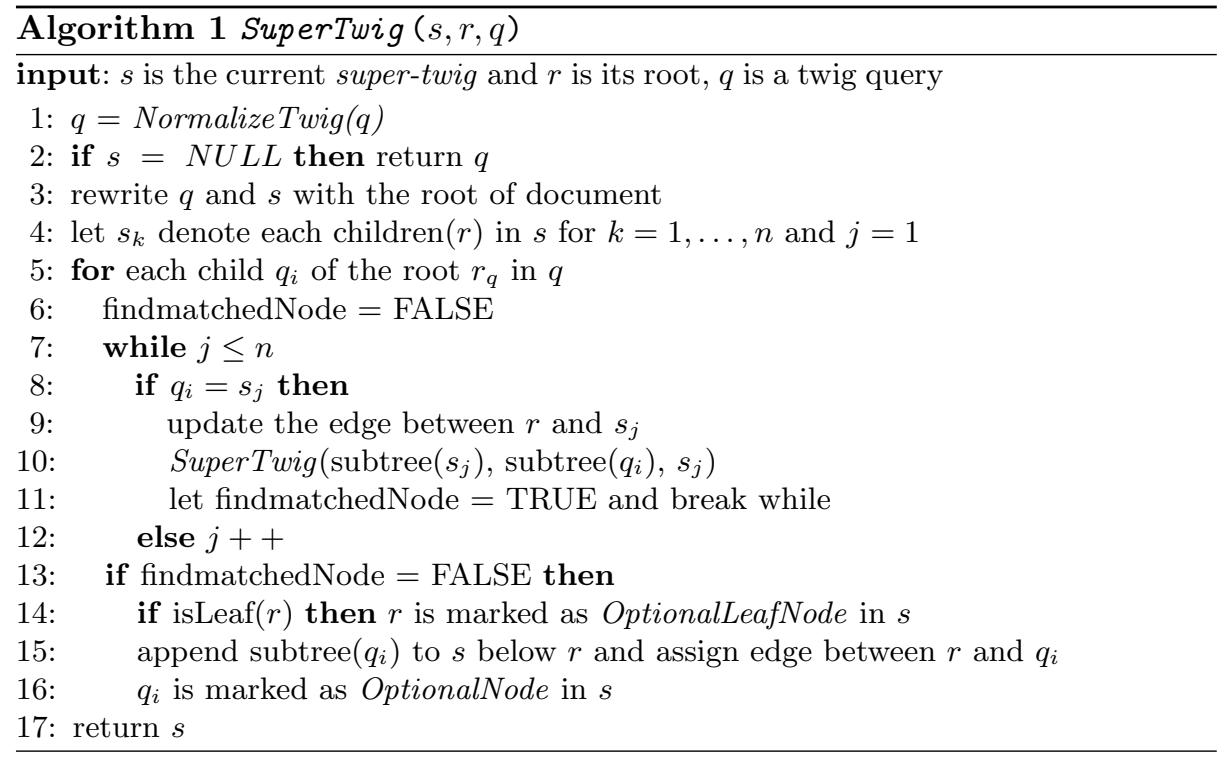




\section{Multiple Twig Queries Matching}

\subsection{Data Structure and Notations}

Let $S Q$ denote the super-twig pattern, and root represent the root node of $S Q$. In our algorithm, each node $q$ in $S Q$ is associated with a list $T_{q}$ of database elements, which are encoded with (start:end, level) and sorted in ascending order of the start field. We keep a cursor $C_{q}$ for each query node $q$. The cursor $C_{q}$ points to the current element in $T_{q}$. Initially, $C_{q}$ points to the head of $T_{q}$. We can access the attribute values of $C_{q}$ by $C_{q}$.start and $C_{q}$. end.

In MTwigStack algorithm, we also associate each query node $q$ in the supertwig query with a stack $S_{q}$. Each data node in the stack consists of a pair: (region encoding of a element from $T_{q}$, pointer to a element in $\left.S_{\text {parent }(q)}\right)$. Initially, all stacks are empty. During query processing, each stack $S_{q}$ may cache some elements and each elements is a descendant of the element below it. In fact, cached elements in stacks represent the partial results that could be further contributed to final results as the algorithm goes on.

\subsection{The MTwigStack Algorithm}

Given the super-twig query $S Q$ of $\left\{q_{1}, \ldots, q_{n}\right\}$ and an XML document $D$, a match of $S Q$ in $D$ is identified by a mapping from nodes in $S Q$ to elements and content values in $D$, such that: (i) query node predicates are satisfied by the corresponding database elements or content values, and (ii) the structural relationships between any two query nodes are satisfied by the corresponding database elements or content values. The answer to the super-twig query $S Q$ with $n$ twig queries can be represented as a set $R=\left\{R_{1}, \ldots, R_{n}\right\}$ where each subset $R_{i}$ consists of the twig patterns in $D$ which match query $q_{i}$.

Algorithm MTwigStack, for the case when the lists contain nodes from a single XML document, is presented in Algorithm 2. We execute MTwigStack(root) to get all answers for the super-twig query rooted at root. MTwigStack operates in two phases. In the first phase, it repeatedly calls the getNext $(q)$ function to get the next node for processing and outputs individual root-to-leaf and root-toOptionalLeafNode path solutions. After executing the first phase, we can guarantee that either all elements after $C_{\text {root }}$ in the list $T_{\text {root }}$ will not contribute to final results or the list $T_{\text {root }}$ is consumed entirely. Additionally, we guarantee that for all descendants $q_{i}$ of root in the super-twig, every element in $T_{q_{i}}$ with start value smaller than the end value of last element processed in $T_{\text {root }}$ was already processed. In the second phase, the function mergeAllPathSolutions() merges the individual path solutions for respective original twig queries.

To get the next query node $q$ to process, MTwigStack repeatedly calls function getNext(root) and the function will call itself recursively. If $q$ is a leaf node of the super-twig, the function returns $q$ without any operation because we need not check whether there exist its descendants matching the super-twig; otherwise, the function returns a query node $q_{x}$ with two properties: (i) if $q_{x}=q$, then $C_{q}$.start $<C_{q_{i}}$.start and $C_{q}$.end $>C_{q_{\max }}$.start for all $q_{i} \in \operatorname{children}(q)$ 


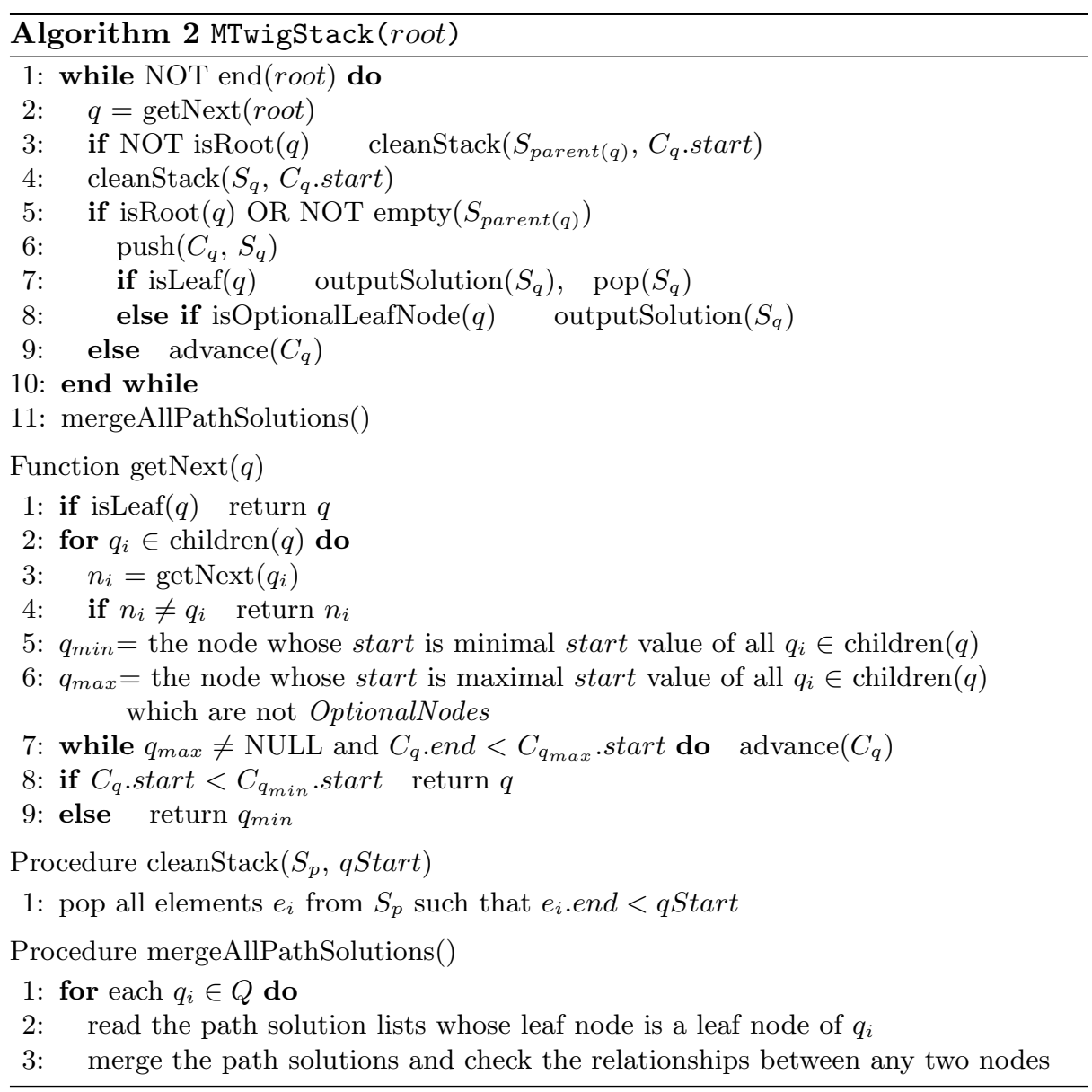

and $q_{i}$ is not OptionalNode (lines 5-8 in Func. getNext $(q)$ ). In this case, $q$ is an internal node in the super-twig and $C_{q}$ will participate in a new potential match. If the maximal start value of $C_{q}$ 's children which are not OptionalNodes is greater than the end value of $C_{q}$, we can guarantee that no new match can exist for $C_{q}$, so we advance $C_{q}$ to the next element in $T_{q}$ (see Figure 2(a)); (ii) if $q_{x} \neq q$, then $C_{q_{x}}$.start $<C_{q_{j}}$.start, for all $q_{j}$ is in siblings of $q_{x}$ and $C_{q_{x}}$.start $<C_{\text {parent }\left(q_{x}\right)}$.start (lines 9 in Func. getNext $\left.(q)\right)$. In this case, we always process the node with minimal start value for all $q_{i} \in \operatorname{children}(q)$ even though $q_{i}$ is OptionalNode (see Figure 2(b)). These properties guarantee the correctness in processing $q$.

Next, we will process $q$. Firstly, we discard the elements which will not contribute potential solutions in the stack of $q$ 's parent (see Figure 2(c)) and execute the same operation on $q$ 's stack. Secondly, we will check whether $C_{q}$ can match the super-twig query. In the case that $q$ is root or the stack of $q$ 's parent is not empty, we can guarantee $C_{q}$ must have a solution which matches the subtree 
rooted at $q$. If $q$ is a leaf node, then it means that we have found a root-to-leaf path which will contribute to the final results of some or all queries; hence, we can output possible path solutions from the node to root; especially, if $q$ is an OptionalLeafNode, we can also output the path for some queries, but we do not pop up $S_{q}$ because $q$ is an internal node and maybe will contribute to other queries in which $q$ is not a leaf node. Otherwise, $C_{q}$ must not contribute any solutions and we just advance the pointer of $q$ to the next element in $T_{q}$ (see Figure 2(d)).

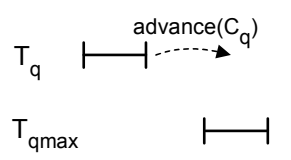

(a) Func. getNext Line 7

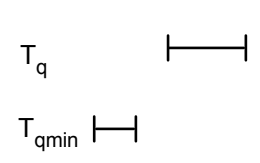

(b) Func. getNext Line 9

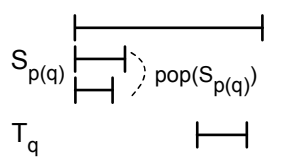

(c) Algo. 2 Line 3

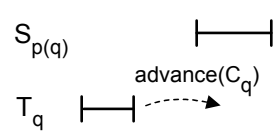

(d) Algo. 2 Line 9

Fig. 2. Possible scenarios in the execution of MTwigStack

In [7], when TwigStack processes a leaf node, it outputs root-to-leaf solutions. However, for super-twig, there are leaf nodes and optional leaf nodes. Different from TwigSack in the first phase, MTwigStack will output path-to-leaf and path-to-OptionalLeafNode solutions if a node $q$ of super-twig is leaf or $O p$ tionalLeafNode (it means $q$ is a leaf node in some queries). Furthermore, in the function $\operatorname{get} N \operatorname{ext}(q), q_{\max }$ is the node whose start is maximal start value of all $q$ 's children which are not OptionalNodes. This restriction guarantees that some nodes in $T_{q}$ are not skipped mistakenly by advance $\left(C_{q}\right)$ when some children of $q$ are not necessary for all the twig queries.

After all possible path solutions are output, they are merged to compute matching twig instances for each twig query respectively. In this phase, we will not only join the intermediate path solutions for each query but also check whether P-C relationships of the queries are satisfied in these path solutions. Merging multiple lists of sorted path solutions is a simple practice of a multiway merge join. In this paper, we do not explain the details for saving space.

MTwigStack is a modification of the TwigStack algorithm. The main diversification is to introduce the concept of OptionalLeafNode, which is treated as a leaf node when processing the super-twig. The algorithm will output intermediate matches when processing the OptionalLeafNodes as they are in fact leaf nodes of some twig queries. Hence, we can easily modify other algorithms such as iTwigJoin [8], TJFast [11], etc.

Example 2. In Figure 3, $S Q$ is the super-twig of $q_{1}, q_{2}$, and $q_{3}$; in $S Q, C$ is an OptionalLeafNode, $D$ and $E$ are OptionalNodes; Doc1 is an XML document. Initially, $\operatorname{getNext}(A)$ recursively calls $\operatorname{getNext}(B)$ and $\operatorname{getNext}(C)$. At the first loop, $a_{1}$ is skipped and $C_{A}$ advances to $a_{2}$ because $a_{1}$ has no descendant node $C$. Then node $B$ is returned and $q=B$. Now the stack $\left(S_{A}\right)$ for parent of $B$ is empty, hence, $b 1$ is skipped and $C_{B}$ points to $b 2$. In the next loop, $A$ is returned and $a 2$ is pushed into $S_{A}$; next, $B$ is returned and $(a 2, b 2)$ is output; then $A$ is returned again and $a 3$ is pushed into $S_{A}$ but $a 2$ will be not popped; $B$ is returned and $b 3$ is pushed into $S_{B},(a 3, b 3)$ and $(a 2, b 3)$ are output. At the sixth 
loop, $C$ is returned and $c_{1}$ is pushed into $S_{C}$. $C$ is an OptionalLeafNode, hence $(a 3, c 1)$ and $(a 2, c 1)$ are output but $c 1$ is not popped. Next $D$ is returned and $d 1$ is pushed into $S_{D}$; Then $F$ is returned, $\left(a_{3}, c_{1}, d_{1}, f_{1}\right)$ and $\left(a_{2}, c_{1}, d_{1}, f_{1}\right)$ are output. Next, $c 2$ is processed, $(a 3, c 2)$ and $(a 2, c 2)$ are output. Finally, $E$ is returned, then $(a 3, c 2, e 1),(a 3, c 1, e 1),(a 2, c 2, e 1)$ and $(a 2, c 1, e 1)$ are output. At the second phase, mergeAllPathSolutions() merges the path solutions of (A, $\mathrm{B})$ and $(\mathrm{A}, \mathrm{C})$ for $q_{1},(\mathrm{~A}, \mathrm{~B})$ and $(\mathrm{A}, \mathrm{C}, \mathrm{D}, \mathrm{F})$ for $Q_{2}$, and $(\mathrm{A}, \mathrm{B})$ and $(\mathrm{A}, \mathrm{C}, \mathrm{E})$ for $q_{3}$. In this phase, we also check whether P-C relationships are satisfied.

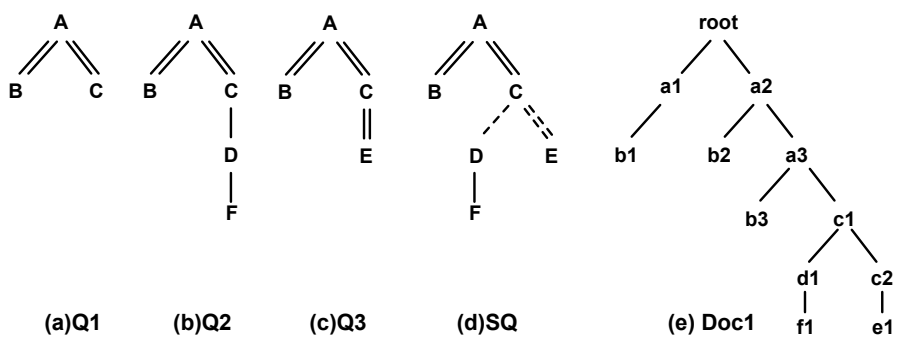

Fig. 3. Illustration to MTwigStack

\section{Experimental Evaluation}

\subsection{Experimental Setup}

We implemented MTwigStack algorithm in Java. All experiments were run on a $2.6 \mathrm{GHz}$ Pentium IV processor with $1 \mathrm{~GB}$ of main memory, running windows XP system. We used the TreeBank [1] and XMark [2] data sets for our experiments. The file size of TreeBank is $82 \mathrm{M}$ bytes, and the file sizes of XMark are $128 \mathrm{~KB}, 2 \mathrm{MB}$, and $32 \mathrm{MB}$ respectively. We test our MTwigStack comparing with TwigStack [7] and Index-Filter [6] with different numbers of queries on these different data sets.

The set of queries consists of 1 to 10000 twig queries, with a random number of nodes between 10 to 20 . The total number of distinct tags in these twig queries is less than $30 \%$ of total distinct tags (75 tags) for XMark data sets, and is less than $15 \%$ of total distinct tags (249 tags) for TreeBank data set.

\subsection{Experimental results}

MTwigStack vs. TwigStack Figure 4 (a) shows the execution time of TwigStack to the execution time of MTwigStack on the four data sets when processing different numbers of queries. We find that whatever the data size is, when there is only one query, these two methods consume the same time; with the number of queries increasing, the processing time increase of MTwigStack is far lower than the increase of TwigStack (e.g. the ratio is about 60 for 1000 queries on the TreeBank data set). This is explained by the fact that MTwigStack process all the multiple queries simultaneously, while TwigStack needs to match the queries one by one. 
In table 1, we show the number of elements scanned by MTwigStack and TwigStack when processing different numbers of queries. Obviously, MTwigStack scans far less elements than TwigStack does. The reason is, for the nodes which appear in multiple queries, MTwgStack scans them only once. But extremely, MTwigStack and TwigStack will scan the same number of elements only when there is no node that appears in all the queries repeatedly, that is, all nodes in the multiple queries are distinct.

MTwigStack vs. Index-Filter We implemented Index-Filter as follows: firstly, decomposing twig pattern into simple path queries for each twig query and combining these path queries into a prefix tree; next, executing the Index-Filter algorithm to get intermediate solutions for each path; finally, joining the path solutions which belong to the same query and eliminating useless solutions.

Figure 4 (b) shows the execution time of Index-Filter to the execution time of MTwigStack. With the increase of data size and number of queries, IndexFilter will run longer time even though it scans the same number of elements as MTwigStack does, as shown in Table 1. The reason is, Index-Filter decomposes a twig query into multiple simple paths during query processing and it will produce many useless intermediate path solutions, as shown in Table 2. Merging more path solutions also need consume more time. Furthermore, Index-Filter also requires more space to keep intermediate results.

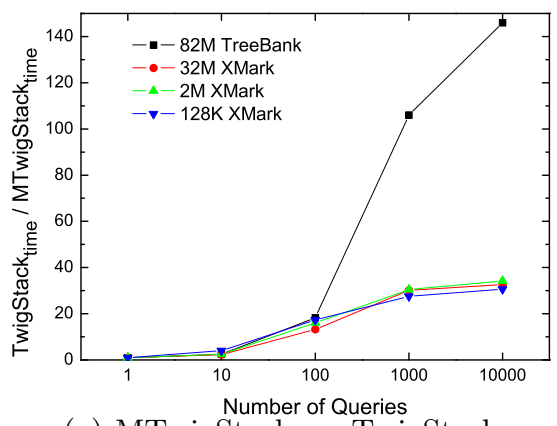

(a) MTwigStack vs. TwigStack

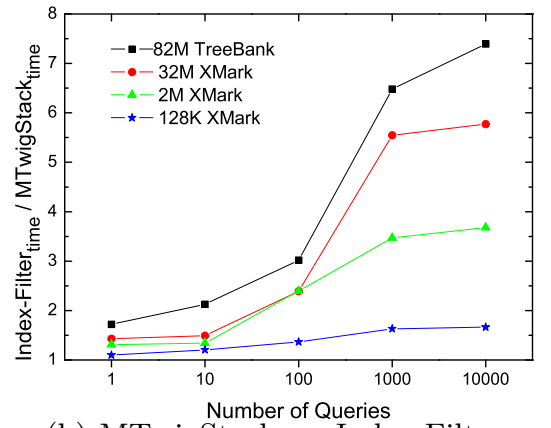

(b) MTwigStack vs. Index-Filter

Fig. 4. Execution time ratio for different data sets

Table 1. The number of scanned elements

\begin{tabular}{|l|r|r|r|r|r|r|r|r|}
\hline Data Set & \multicolumn{2}{|c|}{ 128K XMark } & \multicolumn{2}{c|}{ 2M XMark } & \multicolumn{2}{c|}{ 32M XMark } & \multicolumn{2}{c|}{ 82M TreeBank } \\
\hline No. of Queries & \multicolumn{1}{|c|}{10} & \multicolumn{1}{c|}{100} & \multicolumn{1}{c|}{10} & \multicolumn{1}{c|}{100} & \multicolumn{1}{c|}{10} & \multicolumn{1}{c|}{100} & 10 & \multicolumn{1}{c|}{100} \\
\hline MTwigStack & 286 & 397 & 5027 & 6059 & 78167 & 96357 & 1278766 & 1465232 \\
\hline Index-Filter & 286 & 397 & 5027 & 6059 & 78167 & 96357 & 1278766 & 1465232 \\
\hline TwigStack & 2312 & 18455 & 40337 & 354260 & 635718 & 6005265 & 11685319 & 106760340 \\
\hline
\end{tabular}

\section{Conclusion and Future Work}

In this paper, we proposed a new twig join algorithm, called MTwigStack, to process multiple twig queries with a high structural similarity. Although holistic twig join has been proposed to solve single twig pattern, applying it to multiple 
Table 2. The number of intermediate path solutions

\begin{tabular}{|l|r|r|r|r|r|r|r|r|}
\hline Data Set & \multicolumn{2}{|c|}{ 128K XMark } & \multicolumn{2}{|c|}{ 2M XMark } & \multicolumn{2}{c|}{$32 \mathrm{M}$ XMark } & \multicolumn{2}{c|}{ 82M TreeBank } \\
\hline No. of Queries & 10 & 100 & 10 & 100 & 10 & 100 & 10 & \multicolumn{1}{c|}{100} \\
\hline \hline MTwigStack & 29 & 33 & 349 & 459 & 5401 & 7386 & 646 & 678 \\
\hline Index-filter & 134 & 157 & 2332 & 2827 & 37197 & 44797 & 496691 & 498688 \\
\hline TwigStack & 127 & 1215 & 1237 & 10425 & 19897 & 172360 & 775 & 3965 \\
\hline
\end{tabular}

twig patterns matching is nontrivial. We developed a new concept super-twig with OptionalNode and OptionalLeafNode to determine whether an element is in the shared structure of the XML twig patterns. We also made the contribution by processing the shared structure in the super-twig only once. The experimental results showed that our algorithm is more effective and efficient than the applying TwigStack to each individual twig quires, or applying Index-Filter by decomposing twig queries into many simple path queries.

In the future, we will improve the algorithm based on the following two issues: one is to design an efficient index scheme to fasten the processing speed. Another issue is our method only supports a subset of XPath queries. Some queries, such as //A[B]/C and //D[B]/C, can not be processed efficiently. We will try to process more XPath queries.

\section{References}

1. Treebank. Available from http://www.cis.upenn.edu/treebank/.

2. The xml benchmark project. Available from http://www.xml-benchmark.org.

3. S. Al-Khalifa, H. Jagadish, N. Koudas, J. Patel, D. Srivastava, and Y. Wu. Structural joins: A primitive for efficient XML query pattern matching. In Proceedings of ICDE, 2002.

4. M. Altinel and M. Franklin. Efficient filtering of XML documents for selective dissemination of information. In Proceedings of $V L D B, 2000$.

5. S. Amer-Yahia, S. Cho, L. K. S. Lakshmanan, and D. Srivastava. Minimization of tree pattern queries. In Proceedings of ACM SIGMOD, 2001.

6. N. Bruno, L. Gravano, N. Koudas, and D. Srivastava. Navigation- vs. index-based XML multi-query processing. In Proceedings of ICDE, 2003.

7. N. Bruno, N. Koudas, and D. Srivastava. Holistic twig joins: Optimal XML pattern matching. In Proceedings of ACM SIGMOD, 2002.

8. T. Chen, J. Lu, and T. Ling. On boosting holism in XML twig pattern matching using structural indexing techniques. In Proceedings of ACM SIGMOD, 2005.

9. Y. Diao, M. Altinel, M. Franklin, H. Zhang, and P. Fischer. Path sharing and predicate evaluation for high-performance XML filtering. In ACM Transactions on Database Systems (TODS), volume 28, pages 467-516, 2003.

10. S. Flesca, F. Furfaro, and E. Masciari. On the minimization of xpath queries. In Proceedings of $V L D B, 2003$.

11. J. Lu, T. Ling, C. Chan, and T. Chen. From region encoding to extended dewey: On efficient processing of XML twig pattern matching. In Proceedings of VLDB, 2005.

12. B. Mandhani and D. Suciu. Query caching and view selection for xml databases. In Proceedings of $V L D B, 2005$. 